\title{
Protektionismus und Abschottungstendenzen bremsen und verändern die Globalisierung
}

Schon seit einiger Zeit - und auch bereits vor der CoronaKrise - werden relevante Tendenzen zu einer Verlangsamung und teils auch zu einem Rückbau der Globalisierung diskutiert (Antràs, 2020; Matthes, 2021a; zu den vielfältigen Ursachen vgl. Hüther et al., 2021).

Der Welthandel wuchs in den 2010er Jahren, anders als in den 2000er Jahren, langsamer als die Weltproduktion. Lässt man die Krisen- und Erholungsphasen 2008 bis 2011 und das Krisenjahr 2020 außen vor, so war in den Jahren 2000 bis 2008 die jahresdurchschnittliche Steigerung des Welthandelsvolumens mit 5,2\% laut Angaben des CPB World Trade Monitor fast doppelt so hoch wie das Wachstum der globalen Industrieproduktion. Im gleich langen Zeitraum 2011 bis 2019 hingegen wuchs der preisbereinigte Welthandel mit rund 2,2\% nicht nur wesentlich langsamer, sondern auch weniger dynamisch als die Industrieproduktion, die um rund 2,4\% jährlich zunahm. Zwar ist die Entwicklung des globalen Handels trotz dieser Verlangsamung der Globalisierung in der Tendenz aufwärtsgerichtet, und auch nach dem Einbruch durch die Pandemie findet weltweit so viel internationaler Handel statt wie nie zuvor. Im ersten Halbjahr 2021 war das Volumen des Welthandels um 3,8\% höher als im ersten Halbjahr 2019 und 24,2 \% höher als vor zehn Jahren. Doch es zeichnet sich insgesamt eine geringere Dynamik als in den Jahren vor der globalen Finanz- und Wirtschaftskrise ab.

Das abnehmende Tempo der Globalisierung lässt sich auch an der Verlangsamung des Aufbaus globaler Wertschöpfungsketten beobachten (Fritsch und Matthes, 2020; Kolev und Obst, 2021). Der Anteil der ausländischen Wertschöpfung an den Bruttoexporten sank in den OECD-Mitgliedstaaten nach Angaben der Trade in Value Added Database der OECD von 10,0\% im Jahr 2011 auf nur noch 7,1\% im Jahr 2016 (das letzte von der OECD gemeldete Jahr). Dieser Trend wird bei den drei größten Exportnationen weltweit, China, USA und Deutschland, gleichermaßen beobachtet.

(c) Der/die Autor:in 2021. Open Access: Dieser Artikel wird unter der Creative Commons Namensnennung 4.0 International Lizenz veröffentlicht (creativecommons.org/licenses/by/4.0/deed.de).

Open Access wird durch die ZBW - Leibniz-Informationszentrum Wirtschaft gefördert.
So ist in Deutschland die Bedeutung ausländischer Wertschöpfung sowohl für die inländische Endnachfrage als auch für die Exporte sowohl anteilsmäßig als auch absolut gesunken. Das scheint auf einen Rückbau der Globalisierung in dieser Dimension hinzudeuten. Allerdings lässt sich diese Ansicht relativieren. Denn die Wertschöpfung ausländischer Tochtergesellschaften im Inland ist im selben Zeitraum gemäß Angaben von Eurostat um fast zwei Drittel erheblich gestiegen. Somit ist es nicht auszuschließen, dass ein Teil der zuvor verwendeten ausländischen Wertschöpfung in der Endnachfrage und den Exporten durch die Produktion ausländischer Unternehmen in Deutschland ersetzt wurde, die genau wie der globale Handel nur in einer globalisierten Welt möglich ist. Da jedoch die Tendenz zur Ausweitung der Aktivität ausländischer Unternehmen im Inland nicht neu ist, ist eine abschließende Bewertung ohne genauere Analyse nicht möglich. Festhalten lässt sich aber, dass auch hier in der Gesamtbetrachtung zumindest eine Verlangsamung der Globalisierung zu vermuten ist.

Die globalen Direktinvestitionen waren des Weiteren in den Jahren 2017 bis 2019 nach Angaben der UNCTAD im Trend in absoluten Zahlen rückläufig. Im Jahr 2016 betrugen sie noch über 2 Billionen US-\$, im Jahr 2019 aber nur noch rund 1,5 Billionen US-\$. Selbst im Jahr 2016 war das erreichte Niveau nur wenig höher als im Jahr 2007, als es bei rund 1,9 Billionen US-\$ lag. Auch bei den Portfolio-Investitionen ist ein ähnlicher Einbruch zu beobachten (Antràs, 2020). Zwar bedeutet ein positiver, aber sinkender Wert eine weitere Expansion der globalen Transaktionen, jedoch hat das Tempo auch hier nachgelassen.

Prof. Dr. Galina Kolev leitet die Forschungsgruppe Gesamtwirtschaftliche Analysen und Konjunktur am Institut der deutschen Wirtschaft (IW) in Köln und ist Professorin für VWL an der Hochschule RheinMain in Wiesbaden.

Jürgen Matthes, Dipl.-Volkswirt, leitet das Kompetenzfeld Internationale Wirtschaftsordnung am IW Köln. 
Es lässt sich an dieser Stelle festhalten, dass sich klare Tendenzen zu einer Verlangsamung der Globalisierung zeigen. Im Weiteren wird ein Überblick dazu gegeben, inwieweit wichtige aktuelle und absehbare Tendenzen etwa zu mehr Protektionismus, industriepolitischen Maßnahmen und klimapolitisch begründete Interventionen auch in Zukunft zu einer anhaltenden "Slowbalisation“ (Economist, 2019), also einer verlangsamten Globalisierung beitragen dürften.

\section{Zunahme bei Protektionismus und Handelskonflikten}

Gemäß Zahlen des Global Trade Alerts zeigt sich eine zunehmende Tendenz zum Protektionismus seit der globalen Wirtschafts- und Finanzkrise. So wurden seit November 2008 weltweit mehr als 32.000 Interventionen eingeleitet, die negative Implikationen für den internationalen Handel mit sich bringen. Im gleichen Zeitraum wurden nur knapp 7.000 handelsliberalisierende Interventionen verzeichnet. Der Negativsaldo bei den neuen Handelsbarrieren hat zur relativ schwachen Entwicklung des Welthandels mit beigetragen und so die handelsbezogene Globalisierung ausgebremst.

Diese ohnehin bestehenden Protektionismustendenzen hat die Trump-Administration weiter verschärft, indem sie neue Handelskonflikte und Handelsbarrieren schuf (Kolev und Matthes, 2021). Unter der Biden-Administration dürften diese Probleme, welche die Offenheit im Welthandel gefährden, zumindest etwas kleiner werden.

\section{WTO und Streitschlichtung}

Die Trump-Administration hat das Streitschlichtungsorgan der WTO handlungsunfähig gemacht, indem sie die Berufung von Streitschlichter:innen zur Berufungsinstanz blockierte (Matthes, 2021b). Hinter diesem Schritt stand eine grundsätzliche Skepsis gegenüber der WTO. Die Biden Administration hat hier einen ganz anderen Ton angeschlagen, wie z. B. zwei aktuelle Reden der US-Handelsbeauftragten Katherine Tai im Oktober 2021 dokumentieren (CSIS, 2021; USTR, 2021). Demnach schreiben die USA, die sich auf ihre Funktion als Gründungsmitglied des GATT explizit zurückbesinnen, der WTO eine „sehr, sehr wichtige Rolle" zu (CSIS, 2021). Zwar mahnen sie erheblichen Reformbedarf an, wollen sich dafür aber mit Nachdruck in der WTO engagieren.

Mit Blick auf den Streitschlichtungsmechanismus erkennen sie den Wert dieses Organs an und wollen aktiv mit den anderen WTO-Mitgliedern über eine Reform diskutieren. Nach den Vorstellungen der USA sollen Gegenparts in einem Streitfall wieder stärker Verhandlungslösungen finden, statt Entscheide des Streitschlichtungsorgans zu erzwingen (USTR, 2021). Zudem soll die Berufungsinstanz nicht Regeln setzen, sondern lediglich Regeln auslegen, wie es nach Ansicht der USA anfangs intendiert war. Was das konkret bedeutet und wie sich die USA zu bestehenden Reformvorschlägen positionieren, ist aber noch nicht klar geworden. Daher wird der Streitschlichtungsmechanismus vermutlich noch für längere Zeit außer Kraft sein.

Trotz der schönen Worte der Handelsbeauftragten zur WTO bleibt damit das Problem erhalten, dass Handelsstreitigkeiten durch nicht von der WTO legitimierte Gegenmaßnahmen eskalieren und zu noch mehr Protektionismus führen können.

\section{Handelspolitik von USA, China und der EU}

Die USA, die EU und China kommen zusammen auf über $42 \%$ der globalen Exporte. Zählt man Intra-EU-Exporte noch hinzu, liegt der Anteil bei über $50 \%$. Daher ist es für die Entwicklung der Globalisierung zentral, wie diese Beteiligten in Zukunft agieren werden.

Die drei Hauptaktiven setzen - in unterschiedlichem Maß auf eine gewisse Entkoppelung von der internationalen Arbeitsteilung. Das ist eine wesentliche Veränderung gegenüber der Phase der Hyperglobalisierung (Antràs, 2020).

- In den USA gelten unter Präsident Biden die Schlagworte Made in America und Buy American - mit dem Ziel, die Globalisierung im Zuge von Bidens BuildBack-Better-Agenda angestelltenfreundlicher zu gestalten. Es bleibt aber noch abzuwarten, wie einschneidend diese Maßnahmen in der Praxis sein werden.

- China setzt im Zuge der Dual-Circulation-Strategie mit Nachdruck auf eine mittelfristige Selbstversorgung, um aus geopolitischer Sicht die Abhängigkeit vom Ausland zu mindern. Dies ist bereits in der Praxis spürbar (EUCC, 2021).

- In der EU (und den USA) zeigen sich im Rahmen einer neuen Industriepolitik - in Ansätzen und ausgewählten Bereichen - ähnliche Tendenzen.

Diese Trends werden die Globalisierung weiter verändern, indem der Handel geschwächt, dafür aber die Produktion ausländischer Firmen im Inland weiter gestärkt wird (Matthes, 2021a).

Im Handelskonflikt zwischen den USA und China hat die gegenseitige Zoll-Eskalation zwischen 2018 und 2019 (Matthes, 2020) den bilateralen Handel deutlich gebremst. Die US-Importe aus China waren im Jahr 2019 um $11 \%$ niedriger als im Jahr 2017, während die US-Im- 
porte aus allen anderen Ländern in diesen zwei Jahren um $11 \%$ stiegen. Unter der Biden-Administration gibt es auch weiterhin keine wesentliche Entwarnung, wie die US-Handelsbeauftragte kürzlich deutlich machte (CSIS, 2021). Damit bleiben auf absehbare Zeit rund zwei Drittel der US-Importe aus China mit hohen Zusatzzöllen belegt und auch die chinesischen Gegenmaßnahmen sind weitgehend weiter in Kraft. Möglicherweise kommen USMaßnahmen gegen die Wettbewerbsverzerrungen durch den chinesischen Staatskapitalismus noch hinzu.

Auch die EU ist dabei, ihre Schutzinstrumente gegenüber China zu stärken und zu erweitern (Matthes, 2021c). Damit dürfte auch aus diesem Grund die Handelsverflechtung mit China deutlich langsamer zunehmen als in der Vergangenheit.

Bei den Trump'schen Handelskonflikten mit der EU zeigen sich dagegen Lichtblicke. Positive Entwicklungen sind unter der Biden Administration bei den gegenseitigen Handelsbarrieren im Airbus-Boeing-Konflikt und jüngst auch bei den US-Strafzöllen auf Stahl- und Aluminiumimporte zu verzeichnen. Sehr erfreulich ist auch der Auftakt zu dem von der EU vorgeschlagenen Handels- und Technologierat. Getrübt wird das Bild allerdings durch das Festhalten der USA an dem Phase1-Deal mit China und der damit verbundenen Handelsumlenkung zulasten der EU sowie durch die Verschärfung der Buy-American-Bestimmungen. Insgesamt ist das Glas damit deutlich mehr als halb voll aus Sicht der transatlantischen Beziehungen und der bilateralen Handelsverflechtungen.

\section{Wertschöpfungsketten und neue Industriepolitik}

Der Kurs der Globalisierung hat sich auch während der Pandemie noch weiter verändert und nimmt einen stärker protektionistischen Einschlag, welcher der Internationalisierung noch mehr Schub nehmen dürfte. In den ersten Monaten führte die Verknappung von Medizinprodukten und persönlicher Schutzausrüstung zu einer breiten Diskussion über die Resilienz globaler Lieferketten. Die Diskussion konzentrierte sich sehr stark auf den Status quo und legte ein hohes Gewicht auf die aktuellen Herausforderungen. In vielen Ländern führte dies zu neuen Handelsbarrieren, z.B. in Form von Exportkontrollen oder sogar Exportverboten, insbesondere für Waren, die für den aktuellen Bedarf im Gesundheitsbereich benötigt werden. Auch in Ländern wie Deutschland und den USA, die zu den größten Herstellern von Medizinprodukten und Arzneimitteln zählen, stellte sich die Frage, wie die Versorgung mit solchen lebensnotwendigen Produkten für zukünftige Krisen durch den Aufbau von Produktionskapazitäten und höhere Lagerhaltung gesichert werden kann (vbw, 2021).
Die vorherrschende Knappheit bestimmter Produkte ist im Zuge des Abklingens der Pandemie nicht verschwunden, sondern hat sich auf andere Sektoren verlagert. Die weltweit steigende Nachfrage nach Halbleitern in der Elektronikindustrie aufgrund der Beschleunigung der Digitalisierung während der Pandemie führte zu einem Mangel an diesen wichtigen Zwischenprodukten in anderen Branchen wie der Automobilindustrie. Eine Erhöhung der heimischen Halbleiterproduktion in entwickelten Volkswirtschaften wie den USA und der EU hätte das Problem der starken Übernachfrage ohne fehlgeleiteten Protektionismus aber nicht gelöst, da die steigende globale Nachfrage der ITK-Industrie auch diese Produktion absorbiert hätte und es auch dann zu Engpässen gekommen wäre. Trotzdem begannen die politischen Entscheidungstragenden über Maßnahmen nachzudenken, um die Abhängigkeit von diesem und anderen wichtigen Zwischenprodukten zu senken.

Im Jahr 2021 verabschiedete die Europäische Kommission eine neue handelspolitische Agenda unter dem Leitmotiv der offenen strategischen Autonomie (Europäische Kommission, 2021a). Auch die Stärkung der Widerstandsfähigkeit der Lieferketten (und damit der europäischen Wirtschaft) ist Teil dieser Strategie. Die Resilienz von Lieferketten spielt aber auch in der neuen europäischen industriepolitischen Strategie eine wichtige Rolle (Europäische Kommission, 2021b). Eine Studie im Auftrag der Europäischen Kommission ergab, dass unter den 5.200 untersuchten Produkten bei 34 Schlüsselprodukten zu starke Abhängigkeiten von ausländischen Zulieferungen vorliegt. Dazu zählen insbesondere Rohstoffe und Chemikalien für energieintensive Industrien und das Gesundheitswesen. Die Industriestrategie zielt somit darauf ab, die Krisenresistenz des Binnenmarktes durch ein Überwachungssystem zu verbessern und den Binnenmarkt durch die Digitalisierung der Marktüberwachung zu vertiefen. Darüber hinaus unterstützt die EUIndustriestrategie den Aufbau strategischer Allianzen in Schlüsselsektoren. Unter dem Namen „European Raw Materials Alliance“ wird bereits ein Allianzprojekt im Bereich Rohstoffgewinnung und Seltene Erden mit EU-Mitteln gefördert. Weitere Allianzen sind bereits im Bereich der Produktion von Prozessoren und Halbleitern sowie etwa für Elektromobilität, industrielle Daten, High-End und Cloud Computing geplant.

Solche aufkommenden industriepolitischen Maßnahmen sind nur in wenigen Bereichen berechtigt, da sie die Gefahr eines versteckten Protektionismus bergen und Gegenmaßnahmen wichtiger Handelspartner provozieren können. Deshalb ist es wichtig, mit den Handelspartnern, allen voran mit den USA, ein abgestimmtes Vorgehen zu verfolgen, um keine neue Welle von Handelskonflikten auszulösen (Bown und Irwin, 2021). Wenn dies nicht auf 
breiter Basis gelingt, droht ein industriepolitischer Subventionswettlauf, bei dem die Globalisierung erheblichen Schaden nehmen kann.

\section{Klimaschutz und Nachhaltigkeit in der Handelspolitik}

Jenseits von Protektionismus und Industriepolitik gibt es einen weiteren Megatrend, der den neuen Kurs der Globalisierung prägt. Wie von Parry et al. (2021) berechnet, dürften die weltweiten $\mathrm{CO}_{2}$-Emissionen mit der aktuellen Politik von rund $30 \mathrm{Mrd}$. Tonnen im Jahr 2020 auf $37 \mathrm{Mrd}$. Tonnen bis 2030 steigen, während die Ziele des Pariser Klimaabkommens eine Begrenzung der Emissionen auf etwa $15 \mathrm{Mrd}$. bis $25 \mathrm{Mrd}$. Tonnen voraussetzen. Es gibt mindestens drei große Herausforderungen in Bezug auf Klimaschutz und Nachhaltigkeit, bei denen handelspolitische Maßnahmen nahezu unverzichtbar sind, aber der Globalisierung schaden könnten:

Erstens bedarf es gezielter Maßnahmen, um Carbon Leakage zu stoppen, also Produktionsverlagerungen oder Outsourcing mit dem Ziel, strenge Klimaschutzmaßnahmen zu umgehen, mit dem Effekt, dass auch der $\mathrm{CO}_{2}$-Ausstoß verlagert wird und somit global nicht sinkt. Die EU-Mitgliedstaaten verfolgen bereits eine der strengsten Klimapolitiken weltweit mit einer Tendenz zur weiteren Verschärfung der klimapolitischen Ambitionen. Da andere Länder, meist Entwicklungs- und Schwellenländer, noch andere Herausforderungen zu bewältigen haben und daher längerfristig weniger ambitioniert sein können, wird sich die Kluft zwischen klimabezogenen Regulierungen voraussichtlich weiter vergrößern und damit in der EU weitere Anreize schaffen, Produkte mit höherer $\mathrm{CO}_{2}$-Intensität aus dem Ausland zu importieren. Der am 14. Juli 2021 als Teil des Fit-for-55-Pakets der Europäischen Kommission vorgeschlagene Carbon Border Adjustment Mechanism (CBAM) und das im US-Kongress diskutierte Polluter Import Fee bilden die Grundlage für die Diskussion, um das beschriebene Problem des Carbon Leakage anzugehen.

Zweitens werden handelspolitische Maßnahmen in Erwägung gezogen, um mehr Länder zu einer ehrgeizigeren Klimaschutzpolitik zu bringen. Um die gemäß Parry et al. (2021) nötigen Klimaschutzmaßnahmen zu erreichen, ist aktuell ein umfassender globaler $\mathrm{CO}_{2}$-Preis von schätzungsweise durchschnittlich 75 US- $\$$ pro Tonne $\mathrm{CO}_{2}$ notwendig. Derzeit decken Initiativen zur $\mathrm{CO}_{2}$-Bepreisung nur etwa ein Fünftel der weltweiten $\mathrm{CO}_{2}$-Emissionen ab und der Durchschnittspreis liegt bei nur 3 US- $\$$ pro Tonne $\mathrm{CO}_{2}$. Handelsbezogene Maßnahmen werden seit Jahrzehnten als eine wirksame Möglichkeit angesehen, um mehr Länder an einen Tisch zu bringen (Giersch, 1995), und die Idee des Climate Club von William Nordhaus (2015) wurde in den vergangenen Jahren breit diskutiert und weiterentwickelt (Bardt und Kolev, 2021; Bierbrauer et al., 2021; Kolev, 2021; Paroussos et al., 2021; Tagliapietra und Wolff, 2021). Insgesamt steigt der Druck, $\mathrm{CO}_{2}$-Emittenten, die sich nicht am globalen Klimaschutz beteiligen wollen, durch Handelspolitik zum Umdenken zu bewegen.

Diese beiden Überlegungen bergen allerdings erhebliches Konfliktpotenzial. Dies gilt besonders, wenn es der EU nicht gelingen sollte, einen Klimaklub unter Beteiligung der Industrieländer und wichtiger Schwellenländer wie China zu schaffen, und sie trotzdem Grenzausgleichsmaßnahmen einführen würde. Die dann absehbaren Handelsstreitigkeiten wären breit angelegt und würden die handelsbezogene Globalisierung vermutlich deutlich bremsen.

Drittens hat Nachhaltigkeit auch über den Klimaschutz hinaus in den vergangenen Jahren im handelspolitischen Kontext an Bedeutung gewonnen. Nachhaltigkeitsgesetze und -verordnungen wie das Loi de Vigilance in Frankreich oder das Sorgfaltspflichtengesetz (das sogenannte Lieferkettengesetz) in Deutschland sollen dem Problem der potenziellen negativen Externalitäten durch die Ausnutzung geringerer Arbeits- und Umweltstandards durch heimische Unternehmen im Ausland begegnen. Sie erhöhen jedoch auch die Compliance-Kosten und damit die Handelskosten, insbesondere bei der Beschaffung von Produkten aus Ländern mit niedrigeren Nachhaltigkeitsstandards (Kolev und Neligan, 2021). Es besteht die Gefahr von Handelsumlenkungseffekten weg von Entwicklungsländern, die das Muster der künftigen Globalisierung verändern können.

\section{Herausforderung für deutsches Geschäftsmodell}

Es spricht einiges dafür, dass die geschilderten Entwicklungen und Ansätze die Globalisierung in Zukunft weiter ausbremsen. Aus Sicht der deutschen Wirtschaft mit inrer starken Exportorientierung birgt diese Entwicklung die Gefahr, das deutsche Geschäftsmodell zu untergraben (Matthes, 2021a; Demary et al., 2021). Denn eine geringe Dynamik im Welthandel beschränkt auch die Wachstumsmöglichkeiten für den deutschen Export. Vor allem aber führen der verstärkte Protektionismus, die Madein-America-Strategie der USA und die stärkere Strategie der Selbstversorgung in der EU dazu, dass deutsche Unternehmen mehr Anreize haben, die Produktion in den Zielmärkten anzusiedeln, statt zu exportieren. Das kann mittelfristig die Wertschöpfungs- und Beschäftigungsperspektiven am Standort Deutschland beeinträchtigen.

Ob das Tempo der Globalisierung insgesamt abnehmen wird, ist noch nicht ausgemacht, weil z.B. bei Digitali- 
sierung, Datentransfers und im Dienstleistungshandel Expansionspotenziale liegen, die eine neue Dynamik entstehen lassen könnten. Es erscheint aber fraglich, ob angesichts der protektionistischen Tendenzen ein dafür geeigneter Regulierungsrahmen entstehen kann.

\section{Literatur}

Antràs, P. (2020), De-Globalisation? Global Value Chains in the PostCovid-19-Age, NBER Working Paper, 28115, Cambridge Mass., http:// www.nber.org/papers/w2811 (1. November 2021).

Bardt, H. und G. Kolev (2021), Trade Club for Climate, IW Policy Paper 8/21.

Bierbrauer, F., G. Felbermayr, A. Ockenfels, K. M. Schmidt und J. Südekum (2021), $\mathrm{A} \mathrm{CO}_{2}$-Border Adjustment Mechanism as a Building Block of a Climate Club, Kiel Policy Briefs, 151.

Bown, C. P. und D. A. Irwin (2021), Why Does Everyone Suddenly Care About Supply Chains?, The New York Times, 16. Oktober, 1.

CSIS (Center for Strategic and International Studies) (2021), A Conversation with Ambassador Katherine Tai, U.S. Trade Representatitve, Transcript of event held on October 4, https://csis-website-prod. s3.amazonaws.com/s3fs-public/event/211004_Reinsch_Katherine_ Tai.pdf?o05O9AQ08IkE.W2GCq0_jiObeXAZU_Kr (1.November 2021).

Demary, V., J. Matthes, A. Plünnecke und T. Schaefer (2021), Gleichzeitig: Wie vier Disruptionen die deutsche Wirtschaft verändern, IW-Studie.

Economist (2019), https://www.economist.com/leaders/2019/01/24/thesteam-has-gone-out-of-globalisation (1. November 2021).

EUCC (European Union Chamber of Commerce in China) (2021) Position Paper 2021/2022.

Europäische Kommission (2021a), Trade Policy Review, Directorate-General for Trade, https://trade.ec.europa.eu/doclib/docs/2021/april/ tradoc_159541.0270_EN_05.pdf (19. Mai 2021).

Europäische Kommission (2021b), Europäische Industriestrategie, https:// ec.europa.eu/info/strategy/priorities-2019-2024/europe-fit-digitalage/european-industrial-strategy_de (8. November 2021).

Fritsch, M. und J. Matthes (2020), On the relevance of global value chains and the intra-European division of labour, National Institute Economic Review, Vol. 252, https://www.cambridge.org/core/ journals/national-institute-economic-review/article/abs/on-the-relevance-of-global-value-chains-and-the-intraeuropean-division-oflabour/569A330ECA0D93CBBA439EF1DE80F786 (1. November 2021).

Giersch, H. (1995), Freier Handel und Mindeststandards als ordnungspolitische Herausforderung, Beitrag zur Fachtagung auf der Godesburg, Nach der Reform der Welthandelsordnung: mehr Regeln oder mehr Freiheit für den internationalen Güter- und Dienstleistungsverkehr?
Hüther, M., M. Diermeier und H. Goecke (2021), Erschöpft durch die Pandemie. Was bleibt von der Globalisierung?, Springer Verlag.

Kolev, G. (2021), Trade Club for Climate, WTO of two speeds and International Climate Fund, paper presented at the INFER Annual Conference in Lisboa, Portugal.

Kolev, G. und J. Matthes (2021), Neue handelspolitische Kooperationsmöglichkeiten mit der Biden-Administration, ifo-Schnelldienst, 74(1), 10-13.

Kolev, G. und A. Neligan (2021), Nachhaltigkeit in Lieferketten: Eine ökonomische Bewertung von Gesetzesvorschlägen, IW-Policy Paper, Nr. 5 , https://www.iwkoeln.de/studien/galina-kolev-adriana-neligan-eineoekonomische-bewertung-von-gesetzesvorschlaegen.html (27. Oktober 2021).

Kolev, G. und T. Obst (2021), Global value chains of the EU member states - Policy options in the current debate, IW-Report, im Erscheinen.

Matthes, J. (2020), Die europäische Handelspolitik und China. Schritte zu einer neuen Balance mit fairem Wettbewerb, IW-Analyse, Nr. 138

Matthes, J. (2021a), De-Globalisierung, Protektionismus und Krisen treffen deutsches Exportmodell hart, IW-Kurzbericht, Nr. 52.

Matthes, J. (2021b), How to Unlock the WTO Blockage and Why China Holds the Key, CESifo Forum, 2/2021, März, Vol. 22.

Matthes, J. (2021c), Wettbewerbsverzerrungen durch China. Akademische Evidenz und Ergebnisse einer Befragung deutscher Unternehmen, IW-Report, Nr. 11.

Nordhaus, W. (2015), Climate Clubs: Overcoming Free-riding in International Climate Policy, American Economic Review, 105(4), 1339-1370.

Paroussos, L., A. Mandel, K. Fragkiadikis, P. Fragkos, J. Hinkel und Z. Vrontisi (2021), Climate clubs and the macro-economic benefits of international cooperation on climate policy, Nature Climate Change, 9, 542-546.

Parry, I., S. Black und J. Roaf (2021), Proposal for an International Carbon Price Floor among Large Emitters, IMF Staff Climate Notes, 2021/001, International Monetary Fund.

Tagliapietra, S. und G. B. Wolff (2021), Form a climate club: United States, European Union and China, Nature, Nr. 591, 526-528.

USTR (Office of the U.S. Trade Representative) (2021), Ambassador Katherine Tai's Remarks As Prepared for Delivery on the World Trade Organization, Speech as prepared for delivery, 14. Oktober, https:// ustr.gov/about-us/policy-offices/press-office/speeches-and-remarks/2021/october/ambassador-katherine-tais-remarks-prepareddelivery-world-trade-organization (1. November 2021).

vbw (2021), Internationale Risiken für bayerische Unternehmen, Studie Juni 2021, https://www.vbw-bayern.de/Redaktion/Frei-zugaenglicheMedien/Abteilungen-GS/Wirtschaftspolitik/2021/Downloads/vbw-Studie-Internationale-Risiken-Update-2021-final.pdf (22. Oktober 2021). 